\title{
A Dynamic Packet Scheduling Scheme with Multilevel Priority for Wireless Sensor Network
}

\author{
Ankita A. Chipde \\ P. G. Dept, \\ MBES College of Engineering, \\ Ambajogai, India, 431517.
}

\author{
Veeresh G. Kasabegoudar \\ P. G. Dept, \\ MBES College of Engineering, \\ Ambajogai, India, 431517.
}

\begin{abstract}
Packet scheduling is one of the critical issues in wireless sensor networks. In WSNs most existing scheduling mechanisms use FCFS, non-preemptive or preemptive priority algorithms. However, most of these algorithms incur high processing overhead and high end-to-end transmission delay due to the FCFS concept and starvation of real time and nonreal time data packets in non-preemptive and preemptive priority scheduling algorithms. Also, these packet scheduling algorithms are predetermined and hence cannot change in application requirements of WSNs. Therefore, in this paper, we propose a dynamic multilevel priority (DMP) packet scheduling algorithm to overcome the short comes like starvation of real time data, end-to-end or data transmission delay, and to make the packet scheduling dynamic. It is well known that in DMP scheduling scheme, nodes are organized in hierarchical structure and each node (except those nodes which are located at last level of hierarchical structure) has three level of priority queues in which real time data packets go to highest priority queue and non-real time data packets go to other two queues based on certain threshold levels of their estimated processing time. The DMP scheme proposed in this work was implemented with network simulator (NS2) v.2.32. The results obtained (end-to-end delay, average waiting time, network lifetime, and energy consumption) indicate the proposed DMP scheme is superior to existing first come first serve (FCFS) packet scheduling scheme.
\end{abstract}

\section{General Terms}

Wireless Sensor Network, FCFS.

\section{Keywords}

DMP packet scheduling algorithm, real time data and non-real time data.

\section{INTRODUCTION}

Wireless sensor networks (WSNs) consist of more than hundreds of small spatially distributed autonomous devices using sensors called sensor nodes to monitor the physical and environmental situations such as sound vibration, temperature, pressure, motion and intensity of light [1]. Scheduling is most concentrated term in WSNs because it determines the transmission order of a number of data packets based on their transmission deadline and data priority. For instance, real time data packet should have higher priority to be transmitted to the base station as comparing to non-real time data packet. In WSNs most existing scheduling mechanism uses FCFS [2], non-preemptive, preemptive priority algorithm but these algorithms incur high processing overhead and high end-toend transmission delay [3]. In FCFS scheduling scheme, the data packet schedules based on their arrival times which leads to increased delay for reaching base station (BS). In nonpreemptive packet scheduling schemes, real time data packets have to wait for other already transmitting non-real time data packets to be completed. On the other hand, in preemptive priority scheduling, lower-priority data packets can be placed into starvation for continuous arrival of higher-priority packets but in WSNs sensed data have to reach the BS within specific time period or before the expiration of deadline also real time data should be deliver to BS with minimum end-toend delay. Most existing packet scheduling algorithm are neither dynamic nor suitable for large scale application since these entire schedule are predetermined and static, cannot be changed in response to a change in the application requirements or environments[4]-[6].

Hence, we introduce a dynamic multilevel priority (DMP) packet scheduling scheme for WSNs which overcome all drawbacks occurred in existing scheduling algorithm. The DMP packet scheduling scheme for WSNs, in which sensor nodes are virtually organized into a hierarchical structure. Nodes that have the same hop distance from the BS are considered to be located at the same hierarchical level. Data packets sensed by nodes at different level are processed using TDMA scheme [3]. In dynamic multilevel priority (DMP) packet scheduling scheme for WSNs, where each node maintains three levels into its queue for three different types of data packets. This is because we classify data packets as (i) real time (highest or priority 1), (ii) non-real time remote packets, i.e., packets that arrive from the sensors nodes at lower levels (priority 2), and (iii) non-real time local packets, i.e., the packets that are sensed at the current sensor node (lowest or priority 3). Non-real time data packets are classified based on the location of sensor nodes to balance the end-to-end delay of data packets that are generated at different locations. Non-real time data traffic with the same priority are processed using the shortest job first (SJF) scheduler scheme since it is very efficient in terms of average task waiting time [2]. Preliminaries and related work has been presented in Section 2. Section 3 covers the proposed DMP packet scheduling scheme. Results and analysis of the study are presented in Section 4 followed by conclusions of the work in Section 5.

\section{PRILIMINARIES AND RELATED WORK}

In this section, we present assumption and define some terms which are used in developing dynamic multilevel priority (DMP) packet scheduling scheme.

\subsection{Assumptions}

Following assumptions have been made while implementing the proposed DMP packet scheduling scheme.

- In DMP, data traffic only comprises the real time.

- Data traffic only comprises the incoming data packet is real time or non real time.

- All type (real time/non-real time) of data packets are of same size. 
- $\quad$ Sensor nodes are time synchronized.

- Intermediate node doesn't perform data aggregation for real time data.

- Nodes are located at different levels based on their number of hop counts from base station.

- By using TDMA scheme, timeslots are allocated to nodes, ex: nodes at the lowest level $l_{k}$ are assigned timeslot 1.

- $\quad$ The ready queue at each node has maximum three levels for real time data (pr1), non-real time remote data (pr2) and non real time local data (pr3).

\subsection{Terminology}

Following are the terminologies used in DMP packet scheduling scheme:

- $\quad$ Routing protocol: To achieve high energy efficiency and balance in energy consumption that is increase in the network lifetime among sensor nodes, sensor nodes are grouped together to form zone by using zone based routing protocol $[7,8]$. In zone based routing protocol every zone would have a head, often referred to as a zone head $(\mathrm{ZH})$ and other sensor node become a zone member of that particular zone. All nodes are located in a hierarchical structure based on their hop distance from base station. For example, level 1 and level 2 are decided from the nodes in zones that are one hop and two hops from the base station. In zone based routing protocol, each zone is divided in to a number of small squares in such a way that if sensor nodes exist in a square 1 cover all neighbouring squares. Thus, this protocol reduces the probability of having any sensing hole [9] in the network if the neighbouring squares of the node do not have any sensor node.

- TDMA Scheme: By using TDMA scheme in DMP packet scheduling is performed with variable length time slot. Data packets are transmitted from lower level to base station through intermediate levels that's why node at intermediate and upper levels have more tasks and processing requirements as compared to nodes which are located at lower level. On the other hand real time data packet should not proceed at intermediate level node because they should be delivered to base station with minimum delay that's why real time data have equal and short timeslots at different level.

- Fairness: This Factor ensures that data packets of different priorities get carried out with a minimum waiting time at the ready queue based on the priority of data packets. If any lower priority data packet waits for a long period of time for the continuous arrival of higherpriority data packets, fairness defines a limitation that allows the lower-priority data packet to get processed after a certain waiting time.

- Priority: As discussed above real time data packet should have highest priority and priority of non real time data packet is assigned as per their sensed location. The non real time data packets which are received from lower level have highest priority than the data packet sensed at the node itself.

\subsection{Related Work}

- Lu C. et al. [10] have proposed real-time communication architecture for large scale sensor networks, in which, they use a priority-based scheduling concept. Here, the data packets which travel the longest distance from the source node to base station (BS) but have the shortest deadline is prioritized highest than other data packets. In that, after expiring deadline of particular data packets, they can be dropped at intermediate node. By using this approach, network traffic and data processing overhead reduces, but it is not efficient because it consumes resources such as memory, computation power and increases processing delay.

- In another approach Kambiz Mizanian et al. [11] proposed a RACE - a packet scheduling policy and routing algorithm for real-time large scale sensor networks. In which, they uses loop free Bellman-Ford algorithm to find paths with the minimum traffic load and delay between source and destination, because FCFS scheduling scheme does not work well in real time networks due to the fact that packets have different endto-end deadlines. RACE uses the earliest deadline first (EDF) scheduling concept. In EDF, data packet which have earliest deadline is sent first. In RACE, data packets dropped from priority queue whose deadlines have expired to avoid wasting the network resources but local prioritization at each individual node is not sufficient because packets from different senders can compete against each other for a shared radio communication channel.

- Min Y.U. et al. [12] presented a packet scheduling mechanism that is used in Tiny O. S. [5], [13] - the widely uses operating system of WSNs. In which, packet scheduling scheme classified into non-preemptive (cooperative) or preemptive. Non-preemptive scheduling schemes can be based on a dynamic priority scheduling concept, such as EDF and adaptive double ring scheduling (ADRS) [14] that uses two queues with different priorities. In which, the scheduler dynamically switches between the two queues based on the deadline of newly arrived data packets. If the deadlines of two data packets are different, the shorter deadline data packet would be placed into the higher priority queue and the longer deadline data packet would be placed into the lower priority queue. This packet scheduling scheme of TinyOS are simple and extensively used in sensor nodes but they cannot used for all applications because certain data packets have long execution time and sometime real time data packet placed into starvation.

- To eliminate drawbacks in [12] Zhao Y. et al. [15] proposed an improved priority-based soft real-time packet scheduling algorithm. In which, schedulers traverse the waiting queue for the data packets and select the smallest packet ID as the highest priority to execute. Each data packet is assigned an Execute Counter, EXECUTE_MAX_TIME, i.e., the largest initial task execution time. The management component compares the current packet ID with the previous packet ID. If both are same, the system executes it and decrements the counting variable but the counting variable is null, the management component terminates this packet and other packets get the chance for execution. Data packet priorities are decided during the compilation phase, which cannot be changed during the execution time. If 
high priority data packets are always in execution, the low priority data packets cannot be executed and if lowpriority data packets occupy the resources for a long time, the high priority data packets cannot get response in time.

- However, the work done by Lee E.M et al. [16] proposes a multi-level-queue scheduler scheme which uses different number of queues according to the location of sensor nodes in the network. In this, two kinds of scheduling uses: simple priority based and multi-FIFO queue-based. In the simple priority based, data enters the ready queue according to priority but this scheduling has a high starvation rate. Multi-FIFO queue is divided into maximum three multi queues, depending on the location of node in network.

- In yet another work Karimi E. and Akbari B. [17], reported a priority queue scheduling algorithm for WMSN. In this scheduling scheme, buffer space of intermediate nodes is divided into four queues to hold three different types of video frames and one regular data frames. Data which is placed into first three queues have the highest priority and are scheduled in round-robin fashion and data which is placed into fourth queue is transmitted when the first three queues are empty but these scheduling schemes do not consider variable number of queues based on the position of sensor nodes to reduce the overall end-to-end delay.

All packet scheduling schemes for WSNs discussed in this section are based on various factors like FCFS, preemptive, and non preemptive but are difficult to be dynamically changed. However, dynamic multilevel priority (DMP) packet scheduling scheme proposed in this work is more appropriate for varied WSN applications where both real time and nonreal time data packets are transmitted.

\section{PROPOSED DMP PACKET SCHEDULING SCHEME}

To overcome all the drawbacks of existing scheduling scheme we propose a dynamic multilevel proiority (DMP) packet scheduling scheme. In DMP packect scheduling scheme, all nodes are located into hierachical structure shown in Figure 1. All nodes are located at different level based on their hop distance from base station(BS). Suppose, if some nodes have same hop distance from BS, that is, they are located at same level. All these data packets located at different level are processed using TDMA scheme. By using TDMA scheme, timeslots are assinged to data packets based on their node level. Suppose, nodes that are located at lowest level and second lowest level can be allocated timeslots 1 and 2 .

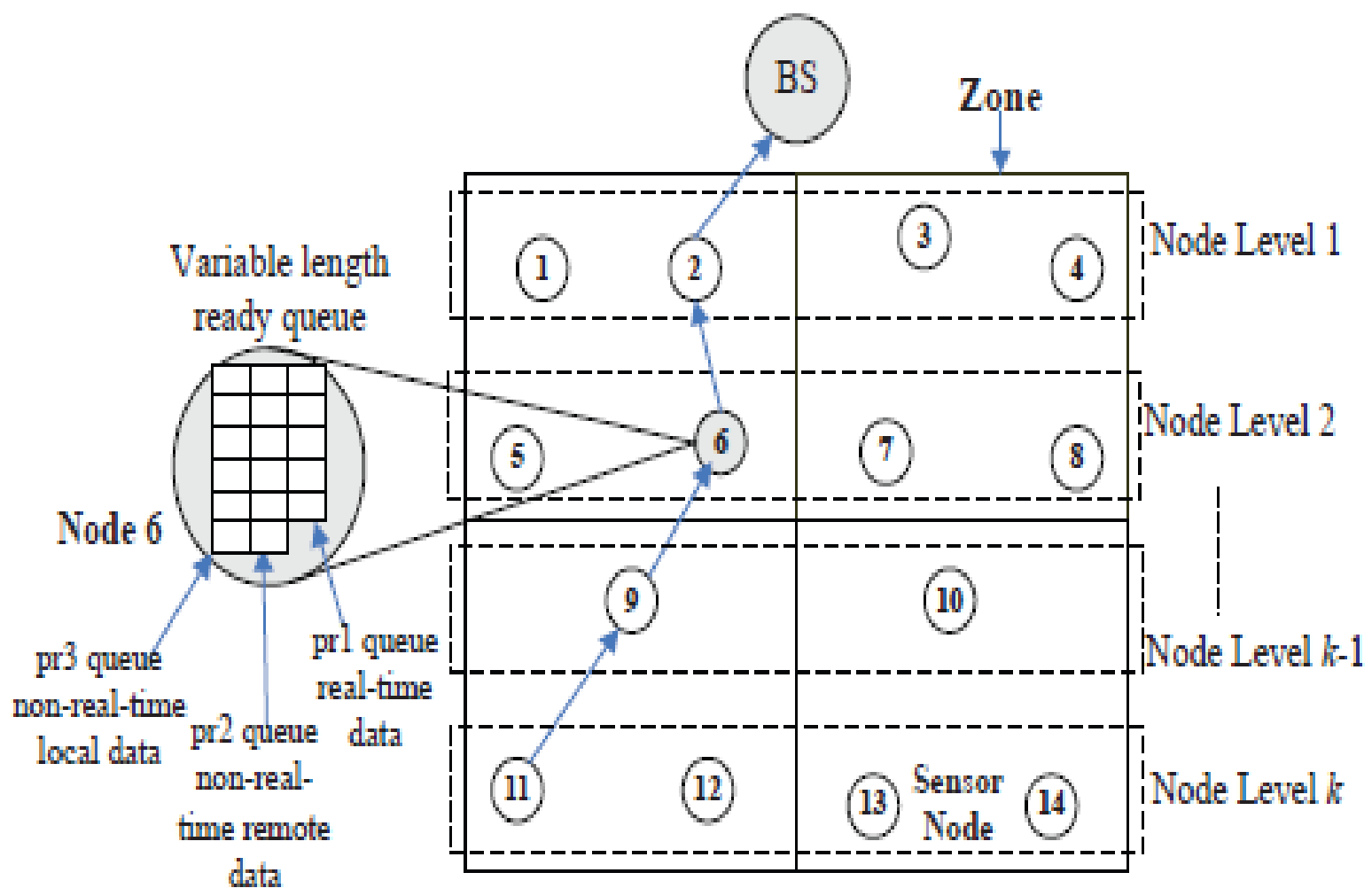

Figure 1: Proposed dynamic multilevel priority (DMP) packet scheduling scheme [3].

In DMP, three level of queues are considered, i.e., ready queues have maximum number of levels is three: priority $1(\operatorname{Pr} 1)$, priority $2(\operatorname{Pr} 2)$, and priorty $3(\mathrm{pr} 3)$. Real time data packets placed into priority $1(\operatorname{Pr} 1)$ queue i.e., real time data packets have highest priority. Non-real time data packets are sensed from lowest level placed into priority $2(\operatorname{Pr} 2)$ queue and finally Non-real time data packet that is sensed from local node itself has lowest priority $(\operatorname{Pr} 3)$. The reason behind that to achieve the overall goals of WSN. Real time data packets which have highest priority are processed in FCFS manner. Every data packet identified by its ID, which consist two parts, namely level ID and node ID. Suppose at the same time two equal priority packet arrive at same ready queue, data packet which is generated at lower level will have higher priority than other packet. This concept reduces end-to-end delay of the lower packet to reach base station(BS). In the 
ready queue, data packets are scheduled among a number of levels that are sensed at a nodes. Node will schedule the packet for transmission according to the priority of the packet and availability of the queue. Due to reduction in packet transmission delay, node can goes to sleep mode as soon as possible, that's why we can improve the energy saving. Node only scheduled priority packet buffering. In our intensification node can check whether expired packets are buffered or not, if buffered then node delets dead packet. Node can drop packet in intelligent manner according to queuing delay. We can reduce buffering delay due to this operation and also we can improve power saving.

\section{RESULT AND ANALYSIS}

The simulation model discussed in earlier paragraphs is implemented in network simulator (NS2 v. 2.32) software. The performance of the proposed dynamic multilevel priority (DMP) packet scheduling scheme against FCFS scheme has been evaluated. The comparison is made in terms of end-toend delay, average waiting time, energy consumption, and network lifetime. All these parameters have been investigated against number of zones. All these parameters are analyzed with network simulator and their performances are presented in Figure 2 to Figure 7. Table 1 presents simulation parameters and their respective values.

Table 1: Simulation parameters and their respective values

\begin{tabular}{|c|c|}
\hline Parameter & Value \\
\hline Number Of Nodes & 101 \\
\hline Number Of Zones & $5-12$ \\
\hline Initial Node Energy & 100 Joule \\
\hline
\end{tabular}

\begin{tabular}{|c|c|}
\hline Transmission Speed & $250 \mathrm{Kbps}$ \\
\hline Network Size & $500 \mathrm{~m}$ X 500 m \\
\hline Propagation Speed & $198 \times 10^{6} \mathrm{~m} / \mathrm{s}$ \\
\hline
\end{tabular}

The end-to-end data transmission delay of real time data packets and combined data packets respectively over a number of zones are presented in Figure 2 and Figure 3. In both cases, it is observed that the proposed DMP scheduling scheme is better than the existing FCFS scheme. This is because of the proposed DMP scheduling scheme real time data packets have highest priority and allows real time data packets to preempt the processing of non-real time data packets, that's why real time tasks have low data transmission delay. Also, in DMP scheme, the tasks which come from the lower level nodes are given higher priority than the packets at the current node. Thus, the average data transmission delay is low.

In another effort, the DMP packet scheduling scheme was implemented in terms of average task waiting time (all types of tasks including real-time data packets) and the same has been depicted in Figure 4 and Figure 5. From these figures (Figure 4 and Figure 5), it can be noticed that DMP packet scheduling scheme is better than FCMS scheme. In another effort we tried to measure and compare network lifetime and energy consumption for all types of data packets.

Figure 6 shows energy consumption of packet scheduling scheme. From the Figure 6 it is clear that DMP packet scheduling scheme is better than FCFS. Figure 7 shows network lifetime of packet scheduling scheme. Again, from the Figure 7 it can be noted that the proposed DMP scheduling scheme has good performance over FCFS.

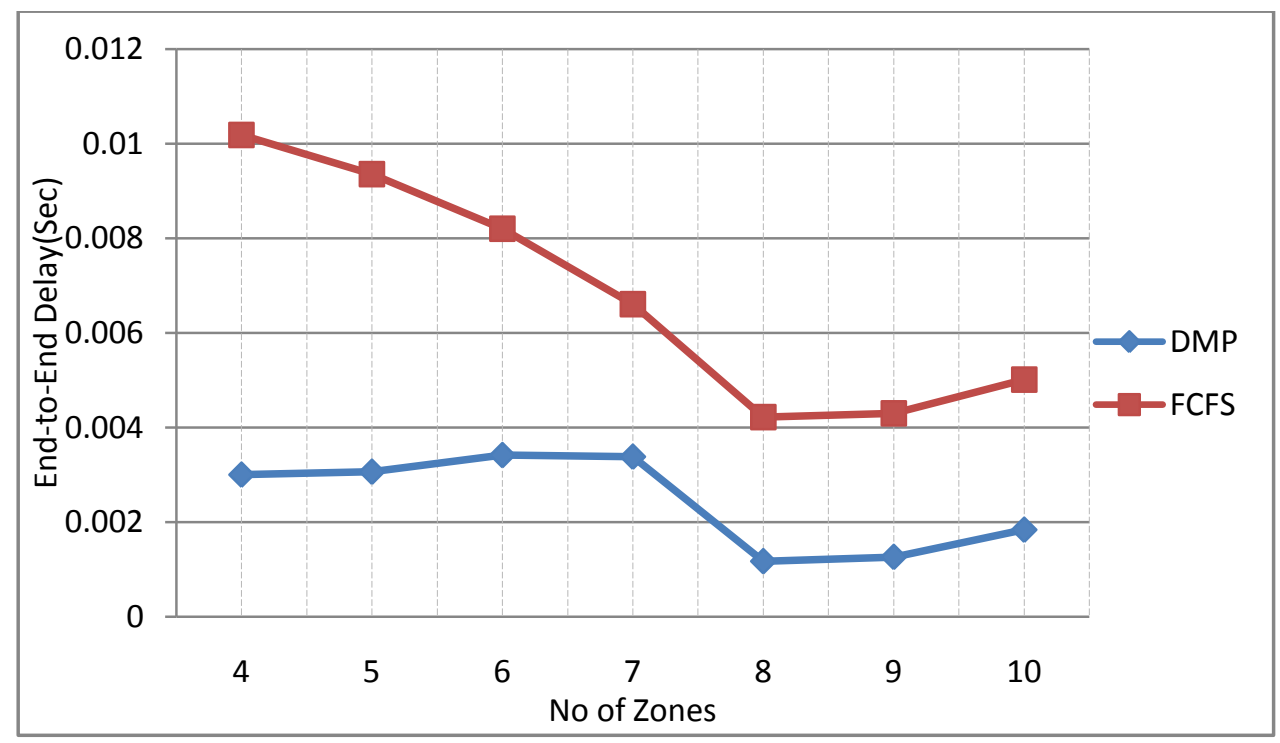

Figure 2: End-to-end delay of all types of data over a number of zones. 




Figure 3: End-to-end delay of real time data over a number of zones.

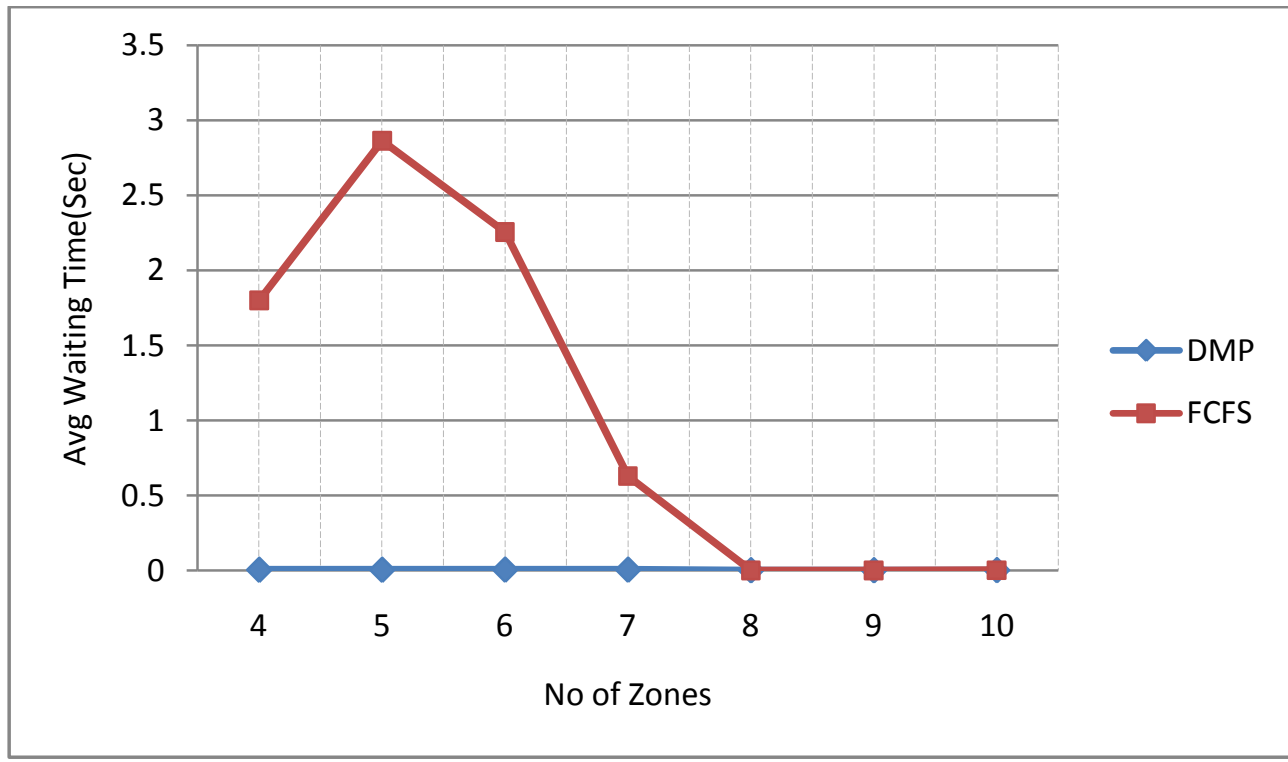

Figure 4: Avg. waiting time of all types of data over a number of zones.

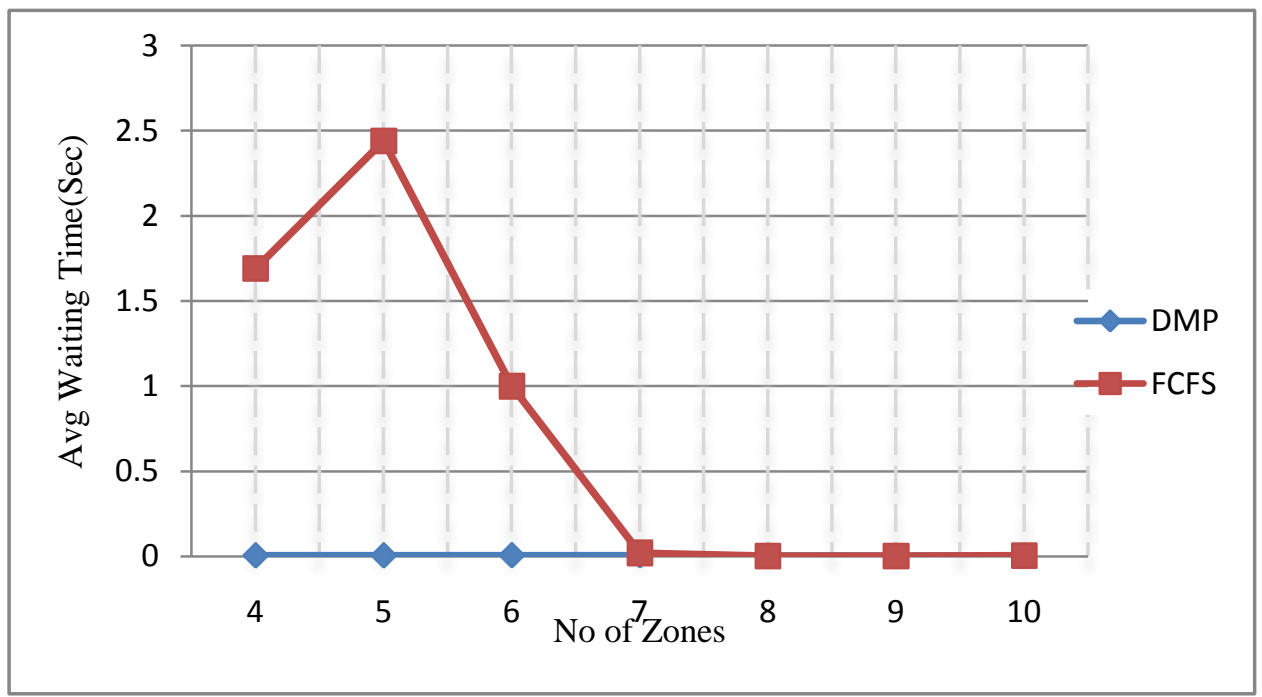

Figure 5: Avg. waiting time of real time data over a number of zones. 


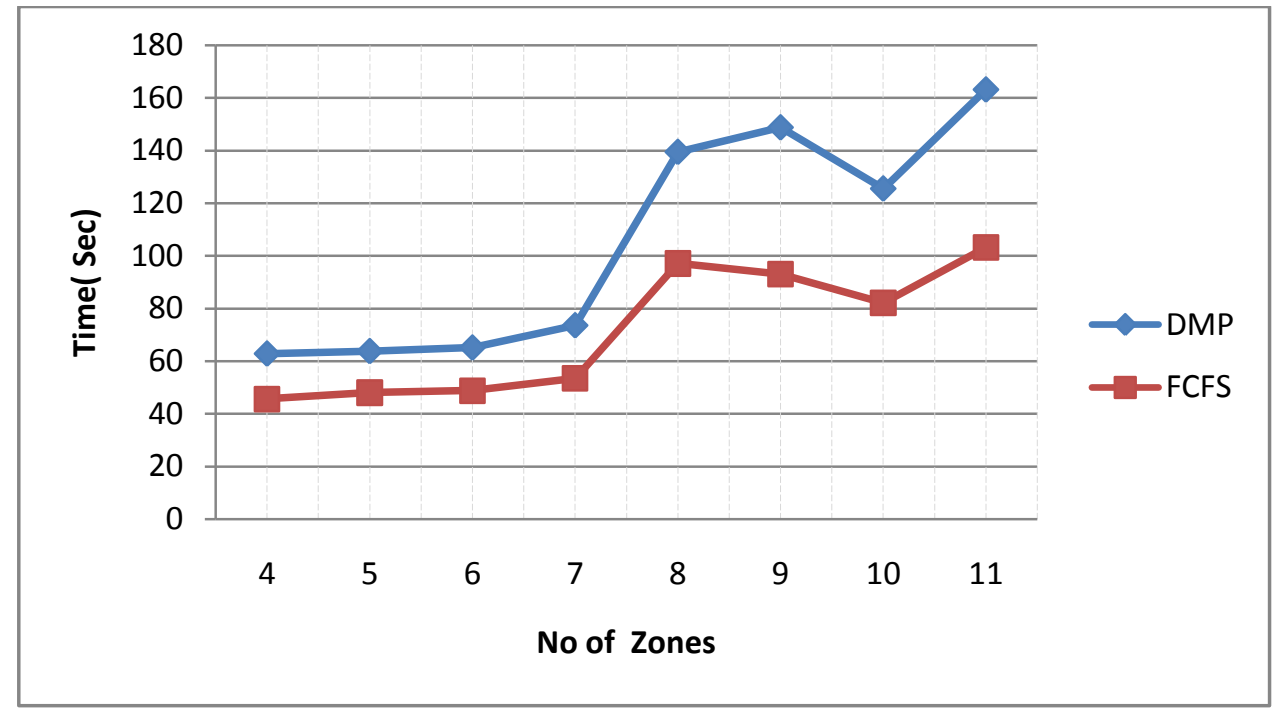

Figure 6 : Network lifetime over a number of zones

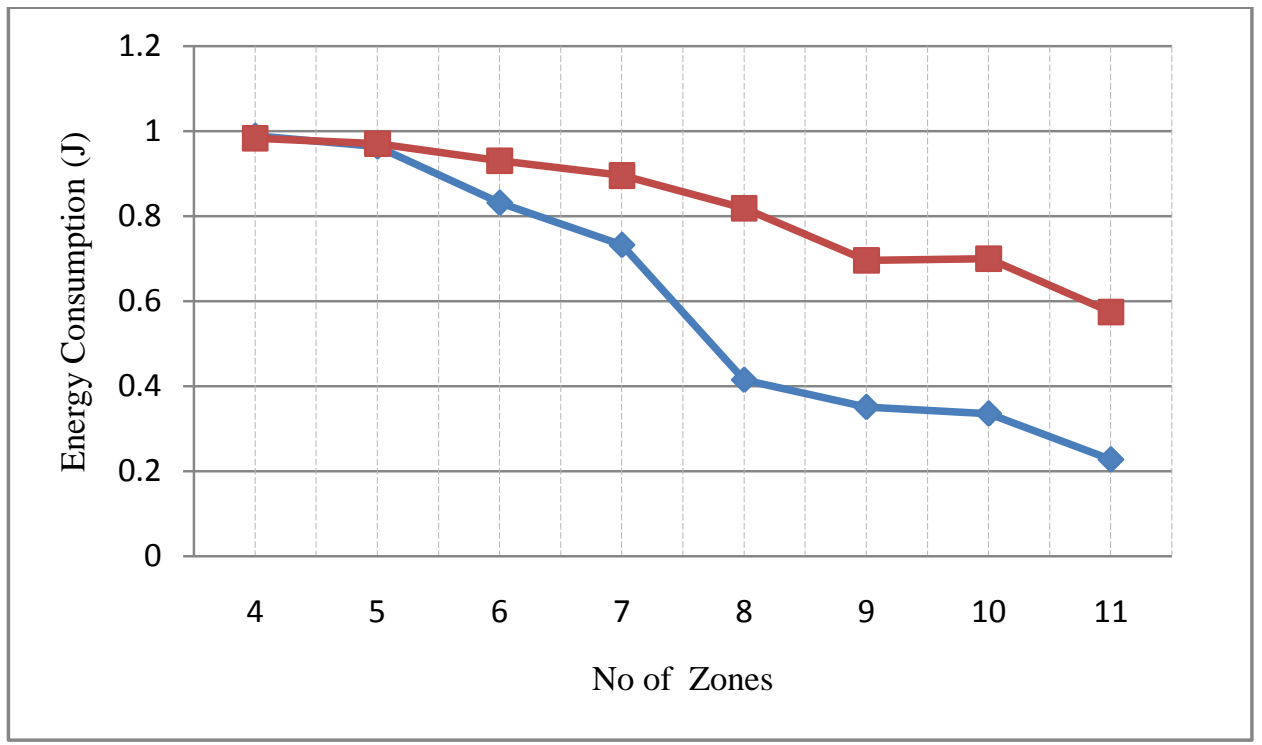

Figure 7: Energy consumption over a number of zones.

\section{CONCLUSION AND FUTURE SCOPE}

Dynamic multilevel priority (DMP) packet scheduling scheme for WSNs has been presented. In DMP, nodes are organized into hierarchical structure, in which, each node except leaf node contains three-level of priority queue to schedule data packets based on their type and priority. By using this concept we tried to minimize the end-to-end delay and the average waiting time. All simulation results presented in this work show that the proposed DMP packet scheduling scheme has better performance than the existing FCFS scheme. The future work of this investigation includes the improvement in assigning priority to data packet that is based on task deadline instead of shortest processing time and also improvement in processing overhead by removing data packets with expired deadline from the medium.

\section{REFERENCES}

[1] Akyildiz, W. Su, Y. Sankarsubramaniam, E Cayirci, "A survey on sensor network," IEEE Common Mag., vol. 40, no. 4, pp. 102-114, 2002.

[2] W. Stalling, Operating System, $2^{\text {nd }}$ edition, Prentice Hall, 1995.
[3] N. Nasser, L. Karim, and T. Taleb, "Dynamic multilevel priority packet scheduling scheme for wireless sensor network," IEEE Transactions On Wireless Communications, vol. 12, no. 4, pp. 1448-1459, 2013.

[4] Y. Zhao, Q. Wang, W. Wang, D. Jiang, and Y. Liu, "Research on the priority based soft real-time task scheduling in TinyOS," International Conf. Inf. Technol. Comput. Sci., vol. 1, pp. 562-565, 2009.

[5] TinyOS. Available: http://webs.cs.berkeley.edu/tos, accessed June 2010.

[6] Available: http://webs.cs.berkeley.edu/tos, accessed June 2010.

[7] S. Chachra and M. Marefat, "Distributed algorithms for sleep scheduling in wireless sensor networks," IEEE International Conf. Robot. Autom., pp. 3101-3107, 2006.

[8] O. Khader, A. Willig, and A. Wolisz, "Distributed wakeup scheduling scheme for supporting periodic traffic in wsns," European Wireless Conf., pp. 287-292, 2009. 
[9] L. Karim, N. Nasser, and T. El Salti, "Efficient zonebased routing protocol of sensor network in agriculture monitoring systems," International Conf. Commun. Inf. Technol., pp. 167-170, 2011.

[10] C. Lu, B. M. Blum, T. F. Abdelzaher, J. A. Stankovic, and T. He, "RAP: a real-time communication architecture for large-scale wireless sensor networks," IEEE Real-Time Embedded Technol. Appl. Symp., pp. 55-66, 2002.

[11] K. Mizanian, R. Hajisheykhi, M. Baharloo, and A. H. Jahangir, "RACE: a real-time scheduling policy and communication architecture for large scale wireless sensor networks," Commun. Netw. Services Research Conf., pp. 458-460, 2009.

[12] M. Yu, S. J. Xiahou, and X. Y. Li, “A survey of studying on task scheduling mechanism for TinyOS,"

International Conf. Wireless Commun., Netw. Mobile Comput., pp. 1-4, 2008.
[13] P. A. Levis, "TinyOS: an open operating system for wireless sensor networks (invited seminar)," International Conf. Mobile Data Manag., p. 63, 2006.

[14] K. Lin, H. Zhao, Z. Y. Yin, and Y. G. Bi, "An adaptive double ring scheduling strategy based on tinyos," $J$. Northeastern University Natural Sci., vol. 28, no. 7, pp. 985-988, 2007.

[15] Y. Zhao, Q. Wang, W. Wang, D. Jiang, and Y. Liu, "Research on the priority-based soft real-time task scheduling in TinyOS," International Conf. Inf. Technol. Comput. Sci., vol. 1, pp. 562-565, 2009.

[16] E. M. Lee, A. Kashif, D. H. Lee, I. T. Kim, and M. S. Park, "Location based multi-queue scheduler in wireless sensor network," International Conf. Advanced Commun. Technol., vol. 1, pp. 551-555, 2010.

[17] E. Karimi and B. Akbari, "Improving video delivery over wireless multimedia sensor networks based on queue priority scheduling," International Conf. Wireless Commun., Netw. Mobile Comput., pp. 1-4, 2011. 